\title{
Pulsar Timing at the Radiotelescope Effelsberg
}

\author{
Ch. Lange ${ }^{1}$, N. Wex ${ }^{1}$, M. Kramer ${ }^{1}$, O. Doroshenko ${ }^{1}$ and D.C. Backer ${ }^{2}$ \\ ${ }^{1}$ Max-Planck-Institut für Radioastronomie, Bonn, Germany \\ ${ }^{2}$ Astronomy Department, University of California, Berkeley, USA
}

\begin{abstract}
We present results of timing observations, obtained at the $100 \mathrm{~m}$-Radiotelescope Effelsberg since 1994. The quality of the pulse arrival times, recorded with the coherent de-disperser EBPP since October 1996, is excellent. Our TOAs are well suited for the measurement of binary parameters of binary pulsars with short orbital periods. Some results are presented.
\end{abstract}

The millisecond pulsar timing-project in Effelsberg started in 1994, using an incoherent filterbank for de-dispersion. Since October 1996 our facilities were augmented by the Effelsberg-Berkeley Pulsar Processor (EBPP), a coherent dedisperser back-end constructed by the Berkeley pulsar group (Backer et al. 1997). For our observations, we use cooled HEMT-receivers with 30 to $45 \mathrm{~K}$ noise temperature at $0.97,1.41$ and $2.7 \mathrm{GHz}$. The exact observing time is stored in the data header by a time stamp from the local H-MASER clock in Effelsberg and off-line corrected to UTC(NIST) using the GPS satellites.

We take typically three integrated profiles per observing session of each source in our timing project. The integration time per profile varies between five and ten minutes. Compact binary systems with binary periods of a few days and less are observed twice per observing session to get arrival times (TOAs) at different orbital phases. For sources that show strong scintillation, as for example PSR J1012+5307, we interactively extend the observing time to use maxima of intensity to record a number of very strong profiles for highest precision TOAs. The spacings between the observing sessions for pulsar timing in Effelsberg are typically a few weeks. However, some sessions are extended to several days in order to cover a full orbit.

For the calculation of the TOA, we use template matching in the Fourier domain (Rawley 1986; Taylor 1992). The error of each TOA is derived from the signal-to-noise ratio of the profiles. It is multiplied by a factor, typically varying between 1.2 and 1.8 to fulfill the $\chi^{2}$-criterion for the post-fit residuals. The statistical behavior of the residuals is tested by comparing their distribution with a Gaussian. In addition, we test the absence of a correlation between subsequent TOAs by combining 2, 4, etc. TOAs and comparing the corresponding rootmean-square residuals. For the evaluation of the TOAs, we use the TEMPOsoftware to get the values for the timing parameters and their estimate errors.

For four pulsars, we observe TOAs with errors of less than one $\mu \mathrm{s}$. For the pulsars J1713+0747 and B1937+21, we even achieve a timing precision up to 200 ns. Timing solutions for ten millisecond pulsars are presented in Table 1. They are in excellent agreement with published parameters (e.g. Toscano et al. 1999). 




1 Pararneters of J1012+5307 were: obtained in collaborution with Jodrell Bank.

Parameters of J1012+5307 wert: ob
it Taken from Toscane et al. (1999)

\begin{tabular}{|c|c|c|c|c|c|}
\hline PSR & $11713+0747$ & $1744-1134$ & $B 1937+21$ & $.32051-0827$ & $.12145-13750$ \\
\hline Right Ascension & $17^{h} 13^{\prime} 49^{\prime r 528644(4)}$ & $17^{h} 44^{\prime} 29^{\prime \prime} 392880(7)$ & $19^{h} 29^{\prime} 38^{\prime \prime} 56011+8(1)$ & $20^{6} 51^{\prime}\left(17^{\prime \prime} 51430(8)\right.$ & $21^{h} 45^{\prime} 50^{\prime \prime} 46711(9)$ \\
\hline Declination & $07^{\circ} 47^{\prime} 37^{\prime \prime} 5319(1)$ & $-11^{\circ} 34^{\prime} 54^{\prime \prime} 5909(7)$ & $21^{\circ} 34^{\prime} 59^{\prime \prime} 13712(3)$ & $-08^{\circ} 27^{\prime} 37^{\prime \prime} 795(7)$ & $-07^{\circ} 50^{\prime} 18^{\prime \prime} 387(4)$ \\
\hline$\mu_{\mathbf{\alpha}}$ (mas/year) & $4.56(7)$ & $18.6(2)$ & - - & & $-15(1)$ \\
\hline$\mu_{\delta}$ (mas/year) & $-4.8(2)$ & $-9(2)$ & . & $\ldots$ & $7(3)$ \\
\hline$\pi$ (mas) & --- & $2.9(6)$ & $\ldots$ & $\cdots$ & - \\
\hline $\mathrm{P}$ (mis) & $4.57013652435089(2)$ & $4.07454587556395(5)$ & $1.557806500606019(6)$ & $4.5086417449715(3)$ & $16.0524236601672(5)$ \\
\hline$\dot{\mathrm{P}}\left(10^{-20}\right)$ & $0.85383(9)$ & $0.8958(5)$ & $10.51083(3)$ & $1.272(2)$ & $2.982(4)$ \\
\hline $\mathrm{DM}\left(\mathrm{pccm}^{-3}\right)$ & $15.9952(2)$ & $3.137(2)$ & $71.0299(4)$ & $20.746(3)$ & $8.983(3)$ \\
\hline Epoch (MJD) & 51000 & 51000 & 51000 & 51000 & 51000 \\
\hline$P_{b}(\mathrm{~d})$ & $67.82512988(1)$ & - & & $0.0991102510(1)$ & $6.838902511(2)$ \\
\hline$x(\mathrm{~s})$ & $32.3424220(1)$ & $\ldots$ & -- & $0.045064(1)$ & $10.164108(1)$ \\
\hline 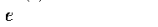 & $0.000074954(6)$ & - & & $\ldots$ & $0.0000195(1)$ \\
\hline $\mathrm{T}_{0}(\mathrm{M} . \mathrm{JD})$ & $51048.016265(1000)$ & - & - & & $51004.422(6)$ \\
\hline$\nu(\mathrm{deg})$ & $176.13666(500)$ & - & & & $200.5(4)$ \\
\hline$\dot{(d e g / \text { year })}$ & - & & ..- & $\ldots$ & . \\
\hline$s_{1}=e \sin \omega$ & - & - & $\cdots$ & . & $-6.8(1) \times 10^{-6}$ \\
\hline$s_{2}=e \cos \omega$ & & - & -- & $\ldots$ & $-18.3(1) \times 10^{6}$ \\
\hline $\mathrm{T}_{\mathrm{Axx}}(\mathrm{MJD})$ & 一 & $\ldots-$ & & $51006.5248768(7)$ & $51006.5248767(7)$ \\
\hline $\bar{P}_{b}\left(10^{-12}\right)$ & - & $\ldots$ & & $-15.4(8)$ & - \\
\hline Number of TOAs & 226 & 67 & 121 & 174 & 80 \\
\hline $\operatorname{RMS}(\mu s)$ & 0.57 & 0.666 & 0.4 & 10.3 & 1.9 \\
\hline
\end{tabular}

Table 1. Timing Results and weighted Residuals, obtianed from Fits to TOAs from Observations at the Radio Telescope Effelsberg

For some binary parameters of short orbital period systems, our values are an improvement. The timing model of PSR J1012+5307, obtained in collaboration with the Jodrell Bank group, is most sensitive for tests of the local Lorentz invariance and gravitational dipole radiation (Wex 2000; Lange et al. in prep.).

\section{References}

Backer D.C., Dexter M.R., Zepka A., Ng D., Werthimer D.J., Ray P.S., Foster R.S., 1997, Publ. Astr. Soc. Pacific, 109, 61

Rawley L.A., 1986, PhD-thesis, Princeton University

Taylor J.H., 1992, Phil. Trans. Roy. Soc. London A, 341, 117

Toscano M., Sandhu J.S., Bailes M., Manchester R.N., Britton M.C., Kulkarni S.R., Anderson S.B., Stappers B.W., 1999, MNRAS, 307, 925

Wex N., 2000, these proceedings 